\title{
A modification of local path marginal cost on the dynamic traffic network
}

\author{
Zheng-feng Huang • Gang Ren • Li-li Lu • \\ Yang Cheng
}

Received: 21 October 2013/Revised: 16 February 2014/Accepted: 19 February 2014/Published online: 11 March 2014

(C) The Author(s) 2014. This article is published with open access at Springerlink.com

\begin{abstract}
Path marginal cost (PMC) is the change in total travel cost for flow on the network that arises when timedependent path flow changes by 1 unit. Because it is hard to obtain the marginal cost on all the links, the local PMC, considering marginal cost of partial links, is normally calculated to approximate the global PMC. When analyzing the marginal cost at a congested diverge intersection, a jump-point phenomenon may occur. It manifests as a likelihood that a vehicle may unsteadily lift up (down) in the cumulative flow curve of the downstream links. Previously, the jump-point caused delay was ignored when calculating the local PMC. This article proposes an analytical method to solve this delay which can contribute to obtaining a more accurate local PMC. Next to that, we use a simple case to calculate the previously local PMC and the modified one. The test shows a large gap between them, which means that this delay should not be omitted in the local PMC calculation.
\end{abstract}

Z. Huang $(\bowtie)$

Faculty of Maritime and Transportation, Ningbo University,

Ningbo 315211, China

e-mail: huang321000@gmail.com

G. Ren · L. Lu

Jiangsu Key Laboratory of Urban ITS, Southeast University,

Nanjing 210096, China

G. Ren · L. Lu

Jiangsu Province Collaborative Innovation Center of Modern

Urban Traffic Technologies, Nanjing 210096, China

Y. Cheng

Department of Civil and Environmental Engineering, University of Wisconsin, Madison, WI 53705, USA
Keywords Transportation network - Path marginal cost . Cumulative flow curve $\cdot$ Dynamic traffic $\cdot$ System optimization

\section{List of symbols}

$Q_{a}^{t} \quad$ The capacity of cell $a$ at time interval $t$

$N_{a}^{t} \quad$ The maximum number of vehicles that can be presented in cell $a$ at time interval $t$

$n_{a}^{t} \quad$ The vehicle occupancy of cell $a$ at time interval $t$

$S_{a}^{t} \quad$ The sending flow from cell $a$ at time interval $t$

$R_{a}^{t} \quad$ The receiving flow to cell $a$ at time interval $t$

$y_{a, b}^{t}$ The transmission flow from cell $a$ to $b$ at time interval $t$

$y_{a}^{t} \quad$ The outflow from cell $a$ at time interval $t$, expressed as $y_{a}^{t}=\sum_{b \in I_{a}^{+}} y_{a, b}^{t}$

$\beta_{a}^{t} \quad$ The vehicle occupancy heading for branch cell $a$ from upstream adjacent diverge cell divided by all the vehicle occupancy in this upstream diverge cell at time interval $t$

$I_{a}^{-} \quad$ The upstream cell set of cell $a$

$I_{a}^{+} \quad$ The downstream cell set of cell $a$

\section{Introduction}

Path marginal cost (PMC) is the change in total travel cost for flow on the network that arises when time-dependent path flow changes by 1 unit. In the fields of transport economy and intelligent transportation, PMC has remained the normally computed value for finding the congestion toll $[1,2]$ or the system-optimal dynamic traffic [3, 4]. However, until now, no method can calculate the marginal cost on all the links after the perturbation of unit vehicle. So generally, different types of local PMC are used to 
approximate the global PMC. Based on cumulative flow curve, Ghali et al. [5] provided a sound analytical formulation for marginal cost on each link along the path where new vehicle was added. These link marginal costs were summed up as a local PMC. However, the link interactions were not considered. Shen et al. [6] proposed a perturbation propagation time method to modify the marginal cost, where the interaction of sequential links was considered. More recently, Qian et al. [7] stated that link interactions on congested diverge links may present a jump-point feature. However, they have not provided an approach to take the jump-point caused delay into the local PMC. Aforementioned definition of PMC can be clearly presented in Fig. 1.

We take Fig. 1 to explain jump-point phenomenon. It is the fluctuation of cumulative vehicles caused by vehicle sequence at link 1 and the rounding calculation procedure for diverging flows. Jump-point phenomenon is related to the dynamic traffic loading method. Generally, simulation methods are selected to load dynamic traffic, because the actual travel time, which is needed to calculate local PMC, can be obtained by traffic simulation. Among them, the cell transmission model (CTM) proposed by Daganzo [8] is relatively an accurate dynamic traffic simulation method, because not only physical queue but also the feature of traffic shockwave is considered. When employing this method to calculate the transmission flow at diverge intersection, a rounding operation is implemented to guarantee the integer formality for flow. If the upstream diverge link is congested, the rounding operation combined with vehicle sequence information could make the delayed vehicle at each time interval not always head for the same downstream branch link as the additional vehicle move toward. For instance, suppose a new vehicle is inserted at the congested upstream cell (a presentation of link segment) at time interval $t$, and the vehicle heading for another downstream branch cell (not the same as the direction of the additional vehicle) is delayed by unit time interval after the rounding calculation for transmission flow. Then, this delay makes the cumulative flow curves in the branch cells lift up (or down) by unit vehicle at time interval $t$. This phenomenon of jumping up and down may continue until the vanishment of upstream bottleneck. Qian et al. [7] names it as a jump-
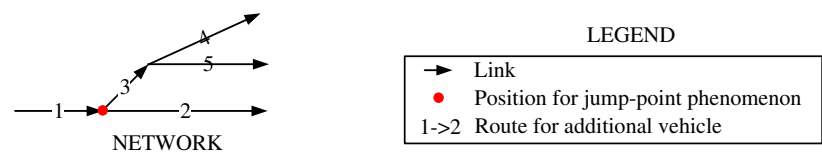

DEFINITION

- Global PMC: Cost influence happens at all the links

- Local PMC at Ghali et al (1995) and Shen et al (2006): Cost influence happens at link 1 and 2

- Local PMC at Qian et al (2012) and this article: Cost influence happens at link 1,2 and 3

Fig. 1 Case chart for the definition of PMC point phenomenon. In their sense, it seems difficult to identify each jump point at the branch cells. Thus, they ignore the jump-point caused delay in the calculation of local PMC. However, it may lead to problems of stability with the application like iterative system-optimal dynamic traffic assignment methods that incorporate calculation of local PMC, such as the method of successive average.

This article provides a modified local PMC for diverge cells. Specifically, calculate its key component, which is the delay generated by jump point. In the first section, CTM at diverge cell is reviewed. In the second section, the calculation of local PMC considering jump-point phenomenon is given. In the third section, using a simple diverge network, we compare the results of the previously local PMC and our modified one.

\section{Review of CTM for diverge cells}

Let the length of each time interval be identical and equal to the free-flow time on each link at diverge intersection. Then, the links in Fig. 2a can be converted to a cell network including three diverge cells in Fig. 2b. Although only two branch links are shown here for convenience, the analysis of more than two branch links is similar.

Because sending and receiving flow, transmission flow, and vehicle occupancy are key variables in the simulation process, we review their formulae at time interval $t$ in advance.

When the backward wave propagation speed is assumed to be the free-flow speed, the formulae for receiving and sending flows of cell $a$ can be described by

$$
\begin{aligned}
S_{a}^{t} & =\min \left\{Q_{a}^{t}, n_{a}^{t}\right\}, \\
R_{a}^{t} & =\min \left\{Q_{a}^{t}, N_{a}^{t}-n_{a}^{t}\right\},
\end{aligned}
$$

where $n_{a}^{t}$ can be obtained from the traffic simulation iteration of previous time interval $t-1$.

The transmission flow formula for flow from cell $a$ to cell $b$ is denoted by

$$
\begin{aligned}
y_{a}^{t} & =\min \left\{S_{a}^{t}, \min \left\{R_{b}^{t} / \beta_{b}^{t} \mid b \in I_{a}^{+}\right\}\right\}, \\
y_{a, b}^{t} & =\beta_{b}^{t} \cdot y_{a}^{t}, \forall b \in I_{a}^{+},
\end{aligned}
$$

If $\beta_{b}^{t}$ is equal to zero, the corresponding term inside the brace should be deleted.

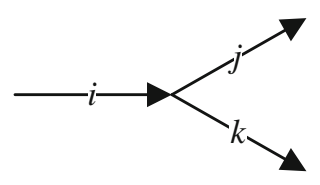

(a) links



(b) cells
Fig. 2 Presentation of diverge cells converted from links 
Equation (3) is used to update the vehicle occupancy of cell $i$ :

$n_{a}^{t+1}=n_{a}^{t}+\sum_{b \in I_{a}^{-}} y_{b, a}^{t}-y_{a}^{t}$.

Note that transmission flow in Eq. (2) should be rounded during each time interval to ensure that the flow and vehicle occupancies are integers.

\section{Calculation of local PMC}

We use the example network shown in Fig. 2 to analyze the local PMC caused by additional vehicle through cell $i$ and $j$. The local PMC is defined to consist of two types of additional cost. One is unmodified marginal cost, which is equal to the previously local PMC that puts the jump-point phenomenon aside; the other is J-P cost, which is specifically used to describe the jump-point caused delay. Qian et al. [7] introduced these two parts. We borrow their theory in the follows. A modification is that the J-P cost trend when uncongested downstream cell is different from their analysis. Finally, we provide the method to attain the J-P cost.

\subsection{Unmodified marginal cost}

We depict unmodified cost according to three types of traffic condition: (1) cell $i$ is uncongested; (2) cell $i$ is congested, and cell $j$ is uncongested; (3) cell $i$ and cell $j$ are congested.

If the first type occurs, the insertion of additional vehicle at cell $i$ will not cause extra delay to its following vehicles. Therefore, the unmodified cost generated for the vehicles at the diverge cells is identical to the travel time of the additional vehicle, which is equal to the free-flow time passing through cell $i$ and $j$.

If the second type occurs, it means that flow perturbation only occurs at cell $i$ not $j$. We depict the unmodified cost generated at cell $i$ here (shown in Fig. 3a). At the beginning of flow-perturbation time interval (the arrival time of additional vehicle), the cumulative arrival flow of cell $i$ lifts up by 1 unit. Until the queue-vanishing time interval $t_{i}^{C}$, can the following vehicles not be influenced by the flow perturbation anymore. Therefore, only those vehicles that arrive among the time range $\left[\tau, t_{i}^{C}\right]$ are delayed by the flow perturbation. Each vehicle is delayed by $l$, which is the inverse of traffic capacity (or discharging rate) at cell $i$. In terms of the whole delayed vehicles $M_{2}-M_{1}$, the total delay time will be $t_{i}^{D}-t_{i}^{B}$. Another component of the unmodified cost is the travel time spent by the added vehicle, which is equal to $t_{i}^{B}-\tau$. Therefore, summing them up can obtain the unmodified cost in cell $i$, which is equal to $t_{i}^{D}-\tau$.
If the third type occurs (shown in Fig. 3a, b), the flow propagation process should be considered. It is required that all the diverge cells should be taken as a whole to calculate the unmodified cost which possesses two components. The first component is the travel time of additional vehicle through cell $i$ and $j$, which is equal to $t_{j}^{B}-\tau$. The second component is the delay for the vehicles $M_{4}-M_{3}$ (Note that $M_{4}-M_{3}$ is part of $M_{2}-M_{1}$ in Fig. 3 although the vertical coordinate intervals may be different for convenience) and $M_{5}-M_{4}$, which is equal to $t_{j}^{D}-t_{j}^{B}$. In other word, we can take cells $i$ and $j$ as a single virtual cell to obtain the unmodified cost which is equal to $t_{j}^{D}-\tau$. Therefore, there is a hidden assumption that no vehicle toward cell $k$ is influenced by the additional vehicle. However, the vehicle sequence information combined with the rounding calculation in the dynamic traffic simulation may make some vehicles toward cell $k$ be delayed, which is a jump-point phenomenon explained in the subsequent subsection.

\section{$3.2 \mathrm{~J}-\mathrm{P}$ cost}

The jump-point phenomenon may occur when cell $i$ is congested. However, the analysis processes for the second and third types are the same; thus, we would only show the analysis for simplicity.

In the first place, we should explain the jump-point phenomenon graphically. When an additional vehicle is added to cell $i$, we should compute Eq. (2) after each time interval to obtain the number of vehicles in the queue of cell $i$ heading for each branch cell. After rounding the number to the nearest integer, we may discover a changed outflow. For instance, it is possible that for a specific time horizon $\left[t^{A}, t^{A^{\prime}}\right]$ of the period following time interval $\tau$, the arrival rate of cell $j$ may lift up (down) by 1 unit, whereas a corresponding decrease (increase) may occur in cell $k$ (shown in Fig. 3). These time horizons are named jump points.

Subsequently, show jump-point phenomenon from the analytical explanation. Suppose that the number of vehicles in queue on cell $i$ heading for cell $j$ during time interval $t$ is denoted as $x_{i, j}^{t}$. So the traffic ratio $\beta_{j}^{t}$ is expressed by

$\beta_{j}^{t}=x_{i, j}^{t} /\left(x_{i, j}^{t}+x_{i, k}^{t}\right)$.

As a special case of Eq. (2), transmission flow is expressed as

$y_{i, j}^{t}=y_{i}^{t} \cdot x_{i, j}^{t} /\left(x_{i, j}^{t}+x_{i, k}^{t}\right)$,
$y_{i, k}^{t}=y_{i}^{t} \cdot x_{i, k}^{t} /\left(x_{i, j}^{t}+x_{i, k}^{t}\right)$.

Suppose that a vehicle at cell $i$ heading for cell $j$ is postponed for unit time interval at time interval $t-1$ when 


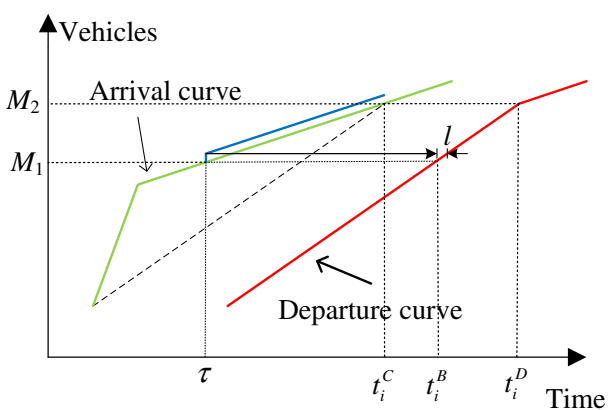

(a) upstream cell $i$

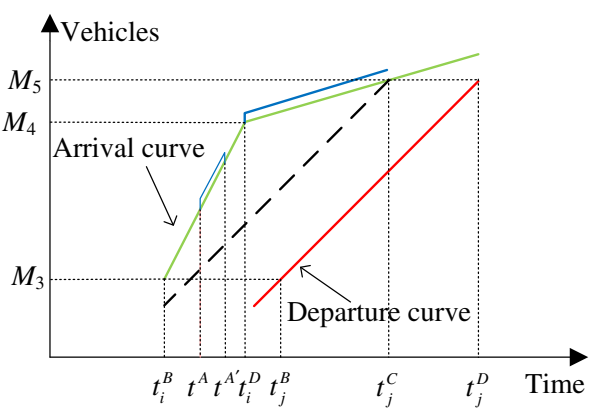

(b) congested downstream cell $j$



(c) congested downstream cell $k$



(d) uncongested downstream cell $k$

Fig. 3 Cumulative flow curves

compared to its previously simulated position. Then, the delayed vehicle will be added to the next time interval. The transmission flow at time interval $t$ can be expressed:

$y_{i, j}^{t}{ }^{\prime}=y_{i}^{t} \cdot\left(x_{i, j}^{t}+1\right) /\left(x_{i, j}^{t}+x_{i, k}^{t}+1\right)$,

$y_{i, k}^{t \prime}=y_{i}^{t} \cdot x_{i, k}^{t} /\left(x_{i, j}^{t}+x_{i, k}^{t}+1\right)$.

Numerically, we round the transmission flow to the nearest integer. In the rounding operation, although some abnormal transmission flow may not make the equation $y_{i, j^{\prime}}^{t}+y_{i, k^{\prime}}^{t}=y_{i}^{t}$ be satisfied, we can avoid this shortcoming by adjusting rounding rule. To describe the jump-point phenomenon clearly, we do not discuss this rounding rule here. If the cumulative flow of cell $j$ or $k$ at time interval $t-1$ is the same with the previously simulated results, but the cumulative flow at time interval $t$ after calculating Eq. (6) is different to the corresponding simulated results, it is indicated that the jump point is generated at time interval $t$. The lifted-down vehicle is postponed to the next time interval $t+1$ and be added to the vehicle occupancy of that time. The above jump point can keep the "jumping" shape if the following transmission flow is unchanged compared to the previously simulated transmission flow at time interval $t+1$. Totally, there could be multiple jump points, which depend on the demands from both paths queued on cell $i$.

We use Fig. 3b, c, d to explain different types of J-P cost. Regarding cell $j$, the unit flow is assumed to be lifted up at time horizon $\left[t^{A}, t^{A^{\prime}}\right]$, so its J-P cost should be added by $t^{A^{\prime}}-t^{A}$. This added part can be explained in other word: a vehicle joins in the queue line of cell $j$ with unit time interval ahead of original time interval, whereas its outflow time is unchanged. Regarding cell $k$, a reduction of one inflow vehicle in the corresponding time horizon is assumed. If cell $k$ is congested, the J-P cost would be decreased by $t^{A^{\prime}}-t^{A}$, whose explanation is similar to the former one. If cell $k$ is not a bottleneck, the J-P cost would be increased by $t^{A^{\prime}}-t^{A}$. The reason is that there is an assumption that the free-flow travel time at cell $k$ cannot be shortened. Therefore, when the vehicles travel through the uncongested cell $k$ with a free-flow travel time, the time interval of outflow will be changed to the same step size with the one of inflow. This could lead to the increase of J-P cost. Undoubtedly, unit time interval would be saved if one inflow vehicle is increased to uncongested cell $k$ during certain time interval. It is noted that the delay modification to uncongested cell $k$ here differs from Qian et al. [7]'s argument which deemed it unchanged.

To obtain the J-P cost during congested time horizon $\left[t_{i}^{B}\right.$, $\left.t_{i}^{D}\right]$, we should prepare the merging queue rule in the upstream intersections in priority. It can obtain the vehicle sequence information or rank the position of each vehicle in the upstream diverge cell, which can help to determine the ratio of the vehicle occupancy from different routes. Assuming that the merging queue rule is given, we can use an analytical method to estimate the J-P cost at the diverge 
Table 1 List of conditions and parameters and J-P cost for downstream cell a time interval

\begin{tabular}{lllllll}
\hline $\begin{array}{l}\text { Condition of } \\
\text { cell } a \in\left\{I_{i}^{+}\right\}\end{array}$ & $\begin{array}{l}\text { Uncongested and } \\
\text { vehicle added }\end{array}$ & $\begin{array}{l}\text { Uncongested and } \\
\text { vehicle erased }\end{array}$ & $\begin{array}{l}\text { Uncongested and } \\
\text { vehicle unchanged }\end{array}$ & $\begin{array}{l}\text { Congested and } \\
\text { vehicle added }\end{array}$ & $\begin{array}{l}\text { Congested and } \\
\text { vehicle erased }\end{array}$ & $\begin{array}{l}\text { Congested and } \\
\text { vehicle unchanged }\end{array}$ \\
\hline$\theta_{t}^{a}$ & -1 & -1 & -1 & 1 & 1 & 1 \\
$\delta_{t}^{a}$ & 1 & -1 & 0 & 1 & -1 & 0 \\
$\begin{array}{l}\text { Trend of J-P } \\
\quad-1\end{array}$ & 1 & 0 & 1 & -1 & 0 \\
\hline
\end{tabular}

Table 2 Comparison of inflow at downstream cells

\begin{tabular}{llllll}
\hline Time interval & $T$ & $T+1$ & $T+2$ & $T+3$ & $T+4$ \\
\hline Inflow at cell $j$ not consider jump point & 3 & 4 & 4 & 4 & 3 \\
Inflow at cell $k$ not consider jump point & 3 & 2 & 2 & 2 & 0 \\
Inflow at cell $j$ consider jump point & 4 & 4 & 4 & 4 & 0 \\
Inflow at cell $k$ consider jump point & 2 & 2 & 2 & 2 & 0 \\
\hline
\end{tabular}

intersection. In the first place, we define two variables. The assignment of these variables and the subsequent J-P cost calculation are described below.

\subsubsection{Congestion level variable}

Assume that $\theta_{t}^{a}$ represents the congestion level of cell $a(\forall a \in$ $I_{i}^{+}$) at time interval $t ; 1$ signifies bottleneck and -1 signifies no bottleneck. The "no bottleneck" also accords to the freeflow speed state in the CTM. Flow profile with respect to density is a trapezoidal pattern, which guarantees that no bottleneck occurs at most of the low occupancy states.

\subsubsection{Perturbation variable}

Assume that perturbation variable $\delta_{t}^{a}$ represents a change in the aspect of cumulative vehicles for cell $a\left(\forall a \in I_{i}^{+}\right)$at time interval $t$. The assigned number $1(0$ or -1$)$ represents 1 vehicle exceeding (no change compared to or 1 vehicle less than) the previously simulated cumulative vehicles. Next, we acquire the perturbation variable time interval by time interval using the information including the perturbation variable and the postponed vehicle at previous time interval and the postponed vehicle at current time interval.

First define $\gamma_{t}^{a}$ to describe the relation between path and cell $a$, and initialize this variable and $\delta_{t}^{a}$ with 0 for different time intervals and cells; then perform the following steps to assign $\delta_{t}^{a}$ beginning from the time interval $t_{i}^{B}$ :

Step 1 Add the previous perturbation variable $\delta_{t-1}^{a}$ to the current perturbation variable $\delta_{t}^{a}$, if $t$ is larger than $t_{i}^{B}$;

Step 2 Identify the route of the new entering vehicle at cell $i$ of the current time interval, which is postponed from the previous time interval or just the additional vehicle we added for the PMC calculation; and assign $\gamma_{t}^{a}$ with 1 if this vehicle is heading for cell $a$;

Step 3 Round the outflow of cell $i$; based on the vehicle sequence in the queue, identify the upcoming postponed vehicle compared to the previously simulated vehicle occupancy; update $\gamma_{t}^{a}$ with $\gamma_{t}^{a}=\gamma_{t}^{a}-1$, if the delayed vehicle is heading for cell $a$;

Step $4 \delta_{t}^{a}=\delta_{t}^{a}+\gamma_{t}^{a}$;

Step 5 let $t=t+1$ and perform sequential steps $1-4$; it will not be completed until the time is out of range $\left[t_{i}^{B}, t_{i}^{D}\right]$.

\subsubsection{Formula for J-P cost}

We use $\sum_{t \in\left[t_{i}^{B}, t_{i}^{D}\right]} \sum_{a \in I_{i}^{+}} \theta_{t}^{a} \cdot \delta_{t}^{a}$ to calculate the J-P cost.

To test the validation of our formula for J-P cost, we will do a comparison here. We list the most probable six cases of cell condition in the first row of Table 1, where different traffic conditions and vehicle variations compared to previously simulated cumulative vehicles are given. The parameters of our method in accord with them can be shown in the middle rows of Table 1 . The trend of J-P cost can be known directly from the cell condition and listed at the last line. Fortunately, their corresponding trend of J-P cost is the same to the one by our analytical formula. Thus, it indicates that our calculation method can attain the accurate J-P cost.

\section{Case study I}

We give a case (shown in Fig. 2) to describe the distinct difference between the previous and modified PMC. Because the jump-point phenomenon may occur only when cell $i$ is congested, whatever the state of downstream cell 


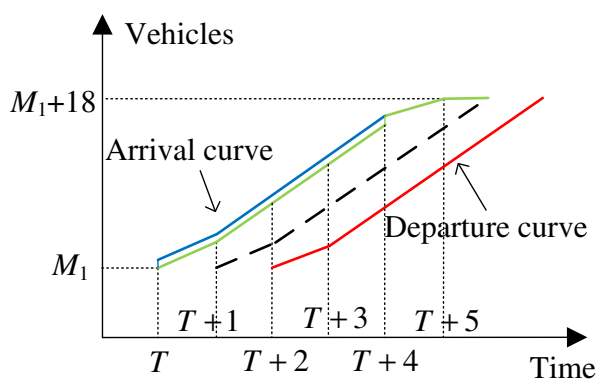

(a) congested cell $j$

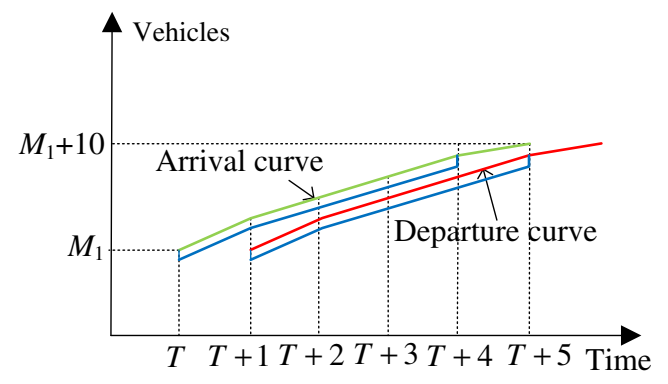

(b) uncongested cell $k$

Fig. 4 Cumulative flow curve when cell $i$ is congested

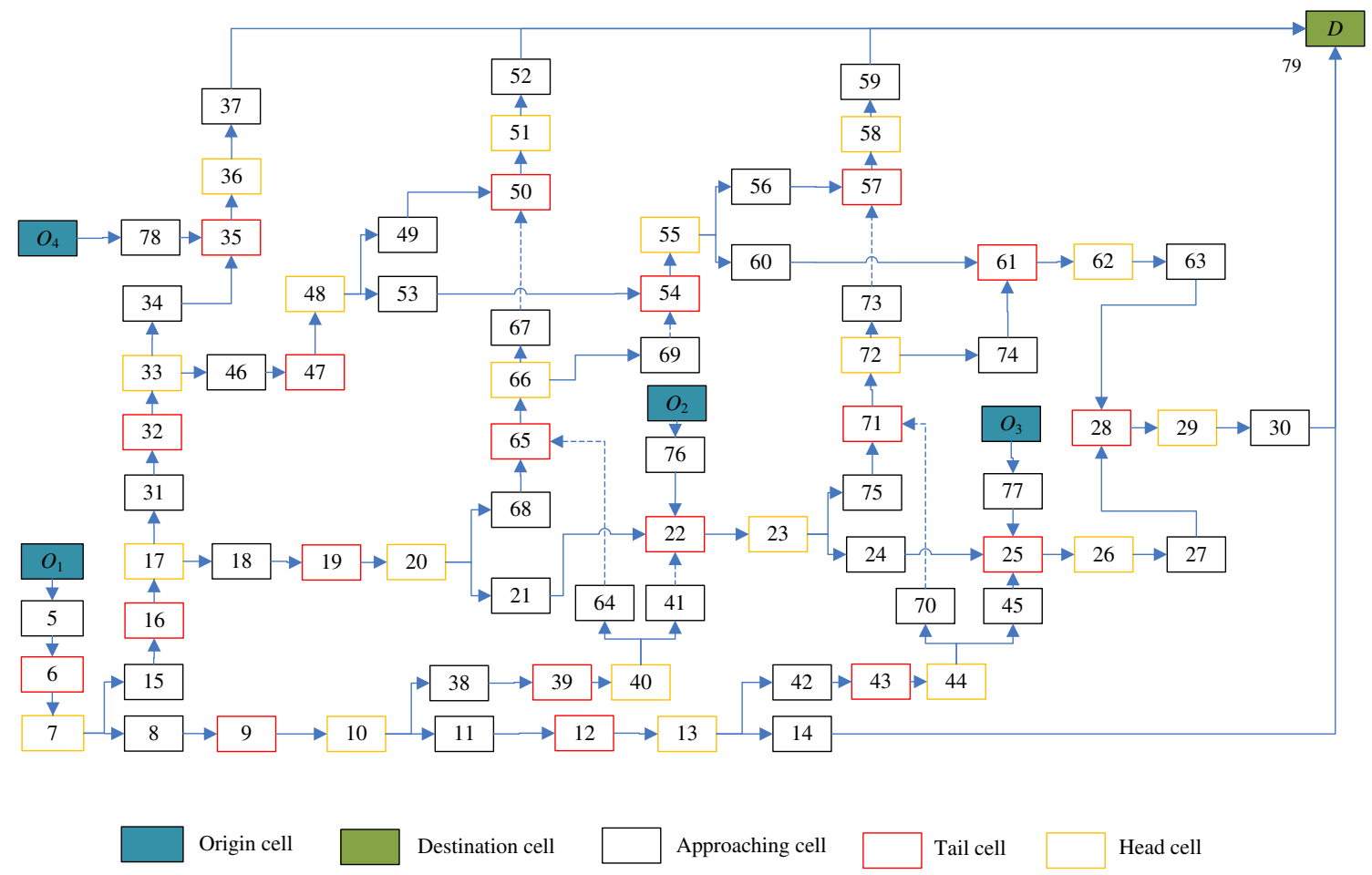

Fig. 5 Cell network

$j$ and $k$. We select the condition that cell $j$ is congested and $k$ is uncongested as an example for clearly explanation. Other conditions are listed as follows.

- The unit time interval is set equal to $3 \mathrm{~s}$;

- The capacity at cell $i$ is set to be six vehicles per time interval;

- The receiving flow of cell $j$ and the maximal vehicle occupancy of cell $i$ are assumed to be large enough to let $y_{i}^{t}=S_{i}^{t}=6$ be possible;

- The vehicle occupancies toward different diverge cells at cell $i$ at $T$ are $x_{i, j}^{T}=5$ and $x_{i, k}^{T}=4$;

- The input flow at cell $i$ toward cell $j$ and $k$ before $T+3$ is always equal to 4 and 2 per time interval separately; then the input flow at cell $i$ is stopped at $T+3$ and the following time intervals;
- Assume that the arrival time interval of additional vehicle at cell $i$ is $T-2$, and use $T$ to replace its departure time interval $t_{i}^{B}$.

If the jump-point phenomenon is ignored, the previously simulated inflows at cell $j$ and $k$ still work. The only difference is that a vehicle is added to the inflow of cell $j$ when the bottleneck is vanished. We can use Eq. (7) to obtain the inflow from $T$ to $T+5$ at cell $j$ and $k$, which are shown in the second and third rows of Table 2.

$y_{i, j}^{t}=S_{i}^{t} \cdot x_{i, j}^{t} /\left(x_{i, j}^{t}+x_{i, k}^{t}\right)$.

Because cell $j$ is congested, we let the travel time of arrival vehicle among the time interval from $T$ to $T+5$ be equal to 2 units. The shape of their flow arrival curves can 


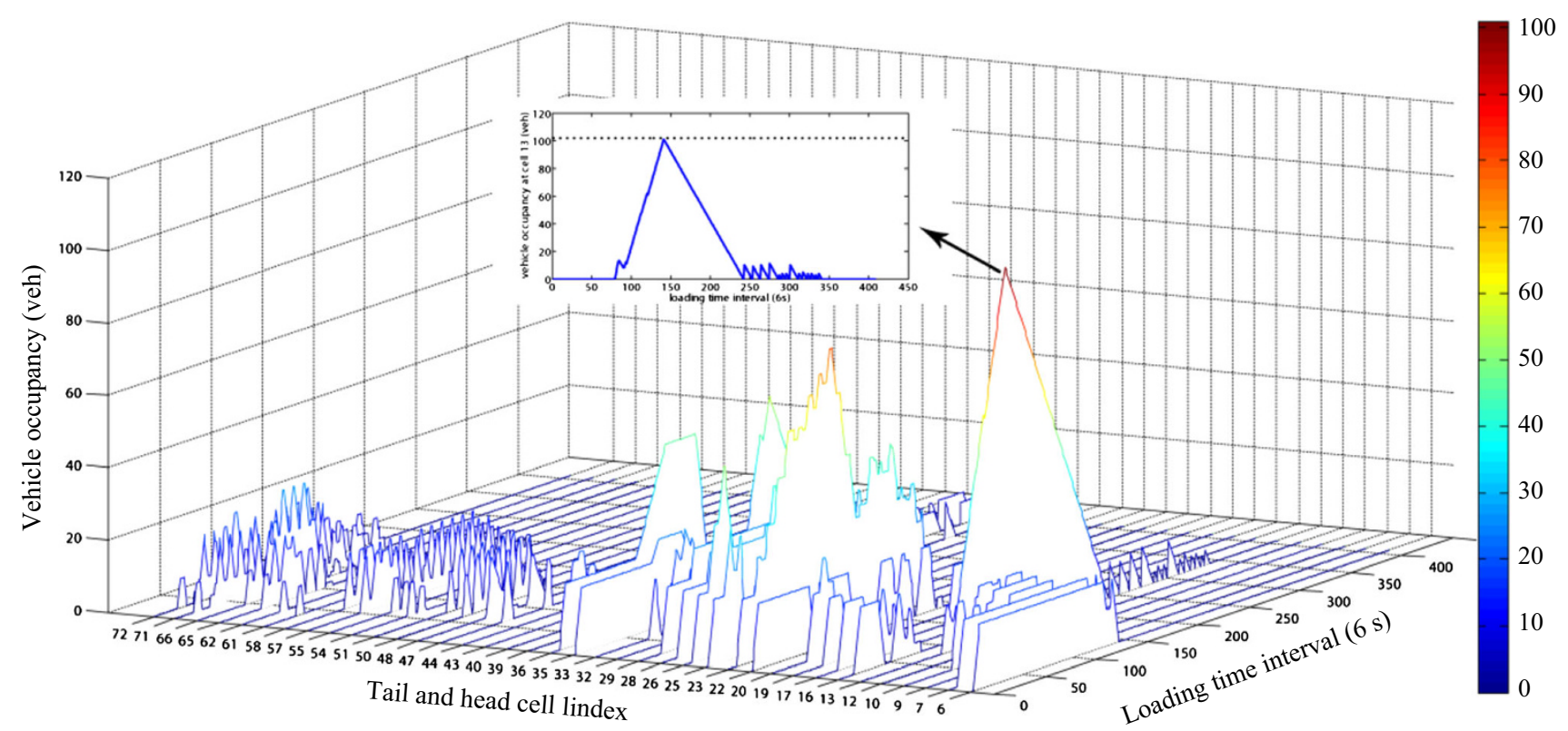

Fig. 6 Vehicle occupancy of tail/head cell changing with time interval

be shown in Fig. 4 (green line). In this case, $T+7$ accords to the time interval $t_{j}^{D}$ of Fig. 4b. So, the previous PMC (or unmodified cost) is equal to nine, which is the subtract result of $T+7$ and $T-2$.

If we consider the jump point, the vehicle occupancy would be $x_{i, j}^{T}=6$ and $x_{i, k}^{T}=4$. After rounding calculation, the inflow is shown in the fourth and fifth rows of Table. 2. The changed curve is depicted with blue line in Fig. 4. It is obviously that the J-P cost is equal to eight. So, the modified PMC is 17. The J-P cost accounts for $47 \%$ of the modified PMC, which should not be ignored.

\section{Case study II}

To illustrate the feasibility of applying the method in larger network Fig. 5, we use the following cell network to calculate the system-optimum dynamic traffic assignment (SO-DTA) by using method of successive averages that embed least PMC searching. Qian et al. [7] demonstrated the feasibility of using this method to solve SO-DTA problem.

Varied cells make up for the network. The origin and destination and approaching cells have the same length with basic cell; the length of other cells equals six basic cells. We assume all the vehicles depart origins cells toward the same destination. The assigned occupancies changing with the time are shown in Fig. 6. No spillback occurs in the cells, indicating that the SO-DTA is reasonable.

\section{Conclusions}

The J-P cost caused by jump-point phenomenon is considered in the calculation of local PMC. An analytical method for solving J-P cost is proposed. This treatment contributes to obtaining a more accurate local PMC. In the first place, based on the historical data and vehicle sequence information, we figure out congestion state and perturbations valuables at each time interval; then, summing up all the products of these two valuables during the whole time intervals can obtain the J-P cost. A comparison of the J-P cost under different cell conditions shows the correctness of our method. A case study shows that the J-P cost may make up a high proportion of local PMC, which should not be ignored.

The application of the modified local PMC in the congestion charging is beyond our study scope. But, it can be realized in theory. For two parallel routes between an OD pair, the actual route costs rather than marginal ones are equal under UE assumption. Theoretically, we can charge toll for the link in the larger PMC route to transfer congested flow to achieve SO network flows as managers desire to see. Congestion toll case with this method in a small network has been tested by Qian et al. [9]. In practice, other newly developed congestion toll related techniques such as tradable credits, flat toll, and tactical waiting $[10,11]$ are more effective, because the ideally timevarying fine toll is hard to solve. However, the comparison of PMC in different routes can still serve as a measurement method for these practical charging types. 
Open Access This article is distributed under the terms of the Creative Commons Attribution License which permits any use, distribution, and reproduction in any medium, provided the original author(s) and the source are credited.

\section{References}

1. De Palma A, Lindsey R (2011) Traffic congestion pricing methodologies and technologies [J]. Transp Res Part C 19(6):1377-1399

2. Zhong R, Sumalee A, Maruyama T (2012) Dynamic marginal cost, access control, and pollution charge: a comparison of bottleneck and whole link models [J]. J Adv Transp 46(3):191-221

3. Rakha H, Tawfik A (2009) Traffic networks: dynamic traffic routing, assignment, and assessment. In: Asakura Y (ed) Encyclopedia of complexity and systems science. Springer, New York, pp 9429-9470

4. Chow AH (2009) Dynamic system optimal traffic assignment-a state-dependent control theoretic approach [J]. Transportmetrica 5(2):85-106
5. Ghali MO, Smith MJ (1995) A model for the dynamic system optimum traffic assignment problem [J]. Transp Res Part B 29(3): $155-170$

6. Shen W, Nie Y, Zhang HM (2006) Path-based system optimal dynamic traffic assignment models: formulations and solution methods. In: Chootinan P (ed) In intelligent transportation systems conference. ITSC'06 IEEE, Toronto, pp 1298-1303

7. Qian ZS, Shen W, Zhang HM (2012) System-optimal dynamic traffic assignment with and without queue spillback: its pathbased formulation and solution via approximate path marginal cost [J]. Transp Res Part B 46(7):874-893

8. Daganzo CF (1995) The cell transmission model, part II: network traffic [J]. Transp Res Part B 29(2):79-93

9. Qian ZS, Michael H (2011) Computing individual path marginal cost in networks with queue spillbacks [J]. Transp Res Rec: Journal of the Transportation Research Board 2263(1):9-18

10. Xiao F, Shen W, Michael Zhang H (2012) The morning commute under flat toll and tactical waiting [J]. Transp Res Part B 46(10):1346-1359

11. Xiao F, Qian ZS, Zhang HM (2013) Managing bottleneck congestion with tradable credits [J]. Transp Res Part B 56:1-14 